\title{
Postsurgical chemotherapy in stage IB nonsmall cell lung cancer: Long-term survival in a randomized study
}

\author{
Mario Roselli $^{1 *}$, Sabrina Mariotti ${ }^{1}$, Patrizia Ferroni ${ }^{2}$, Anastasia Laudisi ${ }^{1}$, Davide Mineo ${ }^{3}$, Eugenio Pompeo ${ }^{3}$, \\ Vincenzo Ambrogi ${ }^{3}$ and Tommaso C. Mineo ${ }^{3}$ \\ ${ }^{1}$ Medical Oncology, Department of Internal Medicine, Policlinico Tor Vergata University, Rome, Italy \\ ${ }^{2}$ Department of Laboratory Medicine and Advanced Biotechnologies, IRCCS san Raffaele, Rome, Italy \\ ${ }^{3}$ Thoracic Surgery, Department of Surgery, Policlinico Tor Vergata University, Rome, Italy
}

\begin{abstract}
Although surgical resection is considered the adequate treatment in early stages of nonsmall cell lung cancer, long-term survival is not satisfactory and recurrence rate is high. We previously showed that postoperative chemotherapy at stage IB reduces recurrences and prolongs overall survival. We extended size and observation period of the study sample and performed a separate analysis for minimally resected patients. The trial was designed as a randomized, 2-armed study with postoperative adjuvant chemotherapy versus surgery alone as control group. All patients had stage IB disease (pT2N0) assessed after a radical surgical procedure (defined as anatomical or minimal). Chemotherapy consisted of cisplatin $\left(100 \mathrm{mg} / \mathrm{m}^{2}\right.$ day 1) and etoposide $\left(120 \mathrm{mg} / \mathrm{m}^{2}\right.$ days $\left.1-3\right)$ for 6 cycles. The primary endpoint was overall survival; secondary endpoint was disease-free survival (DFS). One hundred and forty patients entered the study: 70 were assigned to the adjuvant chemotherapy group and 70 to the control group. Groups were homogeneous for conventional risk factors. There was no clinically significant morbidity associated to chemotherapy. Patients were followed for a mean period of $40.31 \pm 30.86$ months. A significant difference in overall $(p=0.02)$ and disease-free $(p=$ 0.0001) survival was observed between patients undergoing adjuvant chemotherapy vs. control group. Adjuvant chemotherapy significantly improved both overall $(p=0.02)$ and DFS $(p=0.003)$ of anatomically resected patients, but only the DFS $(p=0.02)$ of minimally resected patients. Our results confirm that adjuvant chemotherapy may have a real impact on long-term survival in patients with stage IB nonsmall cell lung cancer being this effect especially evident for those anatomically resected.

(C) 2006 Wiley-Liss, Inc.
\end{abstract}

Key words: adjuvant chemotherapy; NSCLC IB; long-term survival

Radical surgical resection is considered the adequate treatment in providing survival benefits for early nonsmall cell lung cancer (NSCLC), especially for those patients without lymph node involvement. ${ }^{1} \mathrm{~A}$ variety of surgical procedures exist (e.g. standard, atypical, parenchyma-sparing or extended procedures) but all aim at the resection of NSCLC oncologically complete. Notwithstanding, long-term survival is not satisfactory and the recurrence rate is quite high ${ }^{2-4}$ due to the presence of micrometastases ${ }^{5-7}$ not detectable by conventional diagnostic procedures and, therefore, not eradicable by surgery. To prevent this occurrence, postoperative adjuvant systemic therapy was proposed even in the case of surgical procedures deemed radical. ${ }^{8,9}$ A recent meta-analysis of all randomized trials with accrual from January 1965 to December 1991 showed that the absolute risk of death was reduced by $3 \%$ at 2 years and $5 \%$ at 5 years for patients who were treated with postoperative cisplatin-containing regimens. ${ }^{10}$ According to these evidences some institutions, $8,9,11,12$ proposed adjuvant chemotherapy even after presumed radical surgery at stage I, despite discordant results presented by another multicentric study. ${ }^{13}$

On these bases we previously performed a prospective randomized trial including a limited but homogenous subset of NSCLC patients, staged pT2N0 (IB) undergoing anatomical radical resection. ${ }^{14}$ At that time we concluded that adjuvant therapy might reduce recurrences by $\sim 30 \%$ and prolong 5 -year overall survival by $\sim 20 \%$. This article presents the results from the 10-year follow-up analysis of our previous randomized study, ${ }^{14}$ in which the enrollment criteria were extended to include patients who deserved min- imal resection. The purpose of this update was 2-fold: first, to determine whether the reduction of systemic recurrence rates translates into a long-term survival advantage in treated patients that would be reflected in overall survival; second, to establish whether a paradigm shift in IB NSCLC patient management could be confirmed, in which adjuvant chemotherapy after surgery would significantly influence not only locoregional recurrences but also the systemic outcome and, thus, curability.

\section{Methods \\ Study design}

The trial was designed as a monoinstitutional prospective randomized, 2-armed study of postoperative adjuvant chemotherapy versus surgery alone. Patients were enrolled and data collection was performed at the University of Rome Tor Vergata. The study was performed under the approval of the local ethical committee and in accordance with the principles embodied in the Declaration of Helsinki. Each patient signed a fully informed consent before inclusion in the study. The primary endpoint was overall survival; the secondary endpoint was disease-free survival (DFS).

Eligibility criteria for enrollment in the study were:

1. histologically confirmed diagnosis of NSCLC in pathological stage T2NO and absence of viable regional or distant metastatic disease. The staging included clinical history and physical examination, complete blood cell count, serum biochemistry, carcinoembryonic antigen as tumor marker evaluation, urinalysis, plain chest roentgenogram, respiratory functional tests, electrocardiogram, total body computed tomography (CT) scan and fibrobronchoscopy; bone scan was performed only when symptoms were present or alkaline phosphatase level was $50 \%$ greater than the normal limit;

2. age below 75 years with a performance status (Karnofsky index) equal or greater than $90 \%$;

3. beginning of adjuvant chemotherapy within 30 days from surgery;

4. no other organ dysfunction (hepatic, renal and cardiac impairment were evaluated within a maximum period of 6 months preoperatively). Echocardiography and cardiopulmonary stress test were performed when necessary;

5. no history of cancer or radiotherapy or chemotherapy within the last 5 years prior to surgery.

All authors have full access to all data in the study and held final responsibility for the decision to submit for publication. We declare no conflicts of interest.

Grant sponsors: Italian Health Ministry and Tor Vergata University.

*Correspondence to: Medical Oncology, Department of Internal Medicine, University of Rome Tor Vergata, Policlinico Tor Vergata, Viale Oxford, 81, 00133 Rome, Italy. Fax: +3906-20902744

E-mail: mario.roselli@ptvonline.it

Received 23 September 2005; Accepted 18 January 2006

DOI 10.1002/ijc.21933

Published online 20 March 2006 in Wiley InterScience (www.interscience. wiley.com). 


\section{Surgical procedures}

All patients underwent radical surgery. Minimal resection was performed when preoperative forced expiratory volume in $1 \mathrm{sec}$ was equal or lower than $1.3 \mathrm{~L}$ (47 patients). All patients undergoing minimal resection had margins of resection histologically free of tumor invasion; routine mediastinal lymphoadenectomy was performed in all 47 patients. Anatomical resection (93 patients) included posterolateral thoracotomy, pleural cavity exploration, dissection of incidental filmy adhesion of the tumor, routine mediastinal lymph-nodes dissection according to the map of Naruke et al., ${ }^{15}$ venous vessels preparation and ligation prior to arteries and mechanical bronchial suture.

\section{Randomization}

Method to generate random allocation was derived from a computerized software in basic language elaborated by the biostatistics department of our university. The randomization was performed after radical surgery so that the surgical team was not influenced by the random allocation. Furthermore, the sequence was concealed to the oncologist too and they were notified only at the randomization time by telephonic contact with the statistical department.

\section{Adjuvant chemotherapy}

Chemotherapy regimen consisted of 6 courses of cisplatin (CDDP): $100 \mathrm{mg} / \mathrm{m}^{2}$ given on day 1, and etoposide (VP16): $120 \mathrm{mg} /$ $\mathrm{m}^{2}$ at days 1,2 and 3 . Treatment was repeated every 4 weeks. CDDP was infused over $1 \mathrm{hr}$ after intravenous hyperhydratation with 2,000 mL saline plus potassium chloride. VP16 was administrated as a 30 -min intravenous infusion diluted with $250 \mathrm{~mL}$ of saline.

Functional assessment (i.e., complete and differential blood cell counts, urea nitrogen, creatinine clearance, bilirubin and liver enzymes) was repeated before each cycle. Complete and differential blood cell counts were also performed on day 12 to assess hematological toxicity.

Dose reduction and delay in therapy were established according to hematological and nonhematological toxicity. Toxicity was scored according to the World Health Organization toxicity scale (grade $0-4$ ).

In the case of grade 2 hematological toxicity, chemotherapy dosage was reduced to $75 \%$ of the planned dose. In the event of grade 3 hematological toxicity or in case of a creatinine clearance rate lower than $60 \mathrm{~mL} / \mathrm{min}$ the dosage was reduced by $50 \%$. Chemotherapy was not infused if myelotoxicity or neurotoxicity exceeded grade 2 or whether creatinine clearance dropped below $40 \mathrm{~mL} / \mathrm{min}$. The persistence of grade 2 toxicity for a period longer than 14 days implied interruption of treatment.

Incidence and severity of adverse effects of adjuvant chemotherapy were evaluated for each chemotherapic cycle.

Follow up was repeated with the same time-schedule (every 3 months for the first 2 years and then twice a year) for either study arm and included clinical examination, blood biochemistry, serum tumor marker determination (CEA) and chest x-ray. Total body CT-scan was performed every 6 months for the first 2 years and then yearly.

\section{Statistical analysis}

Based on our previously published observations ${ }^{14}$ and assuming a 10 -year survival rate of $40 \%$ for patients at IB stage not receiving adjuvant treatment, we planned to accrue at least 70 patients for each arm of the study. The realized power of detecting a $25 \%$ improvement in overall survival at 10 years using the $5 \%$ level of a conventional 2 -sided test was calculated at $80 \%$. All patients deemed eligible were analyzed on an intention-to-treat basis.

Univariate analysis was performed considering those factors capable of conditioning intergroup differences. Continuous variables were tested by the Mann-Whitney nonparametric test. Dependence among categorical variables was analyzed by the $\chi^{2}$-test or Fisher's exact test when appropriate. Significance level was 0.05. According to the cognitive nature of the study we decided to present the results for patients with a minimum follow-up of 10-year period.

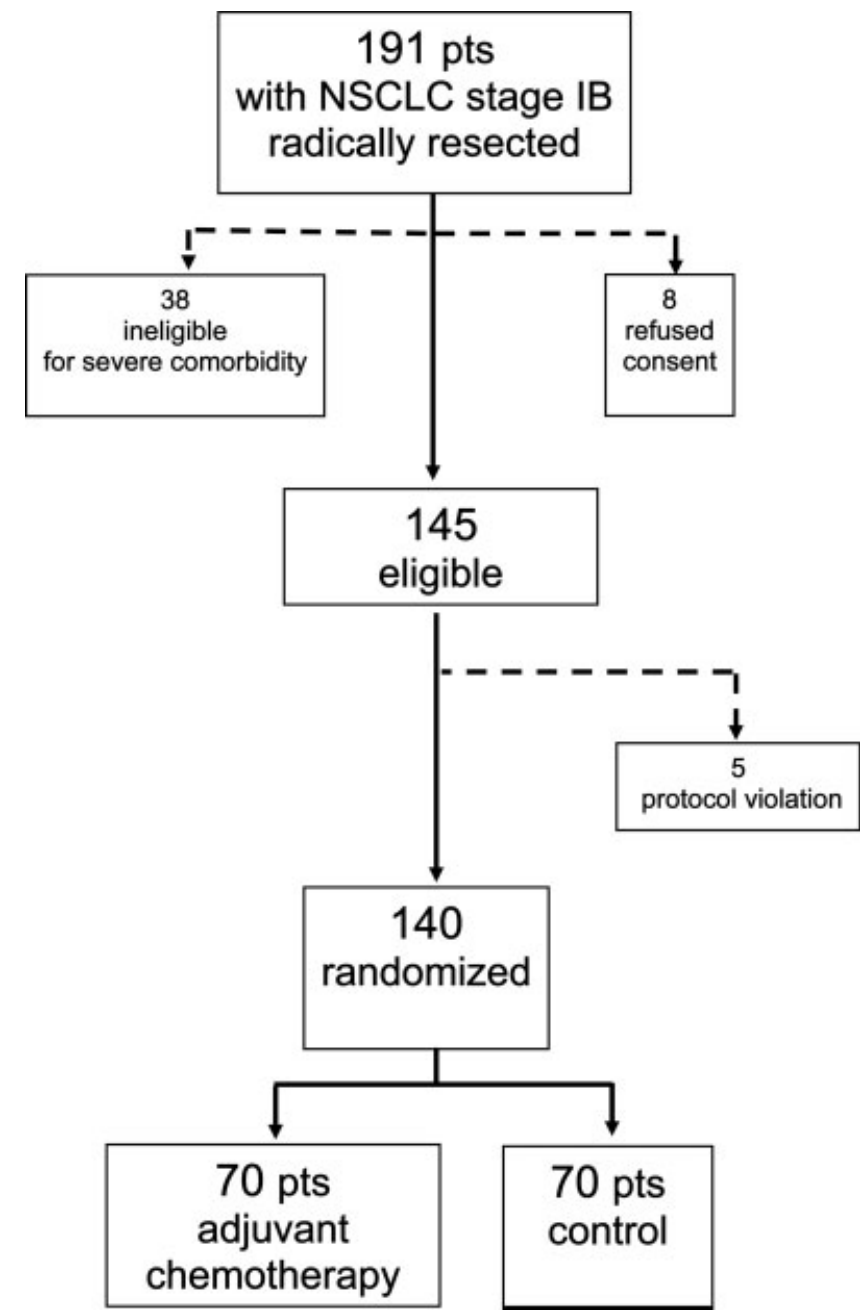

Figure 1 - Trial profile.

Multivariate regression analysis for factors capable of influencing recurrence or death was performed using the Cox's proportional hazards model. ${ }^{16}$ Overall and DFS curves were calculated by the Kaplan-Meier method ${ }^{17}$ and the significance test was assessed according to the Mantel log-rank test. ${ }^{18}$ In all survival analyses, the time to the end point was calculated from the date of surgery of NSCLC until the event date. The following end points were analyzed: (i) DFS was defined as the interval between the date of diagnosis and the date of the first NSCLC recurrence; with the event being any recurrence, locoregional or systemic; (ii) overall survival was defined as the interval from date of diagnosis until the date of malignancy-related death. All calculations were made using computer software packages (Systat, version 9.0, SPSS, Chicago, IL).

\section{Results}

Of 191 patients considered for randomization, 46 were judged ineligible due to severe comorbidity, delayed recovery after surgery or unwillingness to provide informed consent. Additional 5 patients were excluded after randomization because of major protocol violation.

A total number of 140 patients (117 men and 23 women), with pathological stage IB of NSCLC were judged eligible and allowed into the study from January 1988 to December 1994. Seventy patients were randomized to each study arm. Figure 1 shows the trial profile and the patients' characteristics are summarized in Table I. 
TABLE I - PATIENTS CHARACTERISTICS AND BALANCE OF RISK FACTORS

\begin{tabular}{|c|c|c|c|c|c|c|}
\hline & $\begin{array}{c}\text { Anatomical resection } \\
(n=93)\end{array}$ & $\begin{array}{c}\text { Minimal resection } \\
(n=47)\end{array}$ & $p$-value & $\begin{array}{c}\text { Adjuvant } \\
(n=70)\end{array}$ & $\begin{array}{c}\text { Control } \\
(n=70)\end{array}$ & $p$-value \\
\hline Age $($ mean $\pm \mathrm{SD})$ & $61.8 \pm 9.8$ & $67.4 \pm 7.7$ & n.s. & $64.7 \pm 9.9$ & $62.9 \pm 9.2$ & n.s. \\
\hline Sex (male $v s$. female) & 80 vs. 13 & 37 vs. 10 & n.s. & 64 vs. 6 & 53 vs. 17 & n.s. \\
\hline Histology (nonsquamous vs. squamous) & 43 vs. 50 & 22 vs. 25 & n.s & 31 vs. 39 & 30 vs. 36 & n.s \\
\hline Site (central $v s$. peripheral) & 55 vs. 38 & 20 vs. 27 & n.s & 43 vs. 27 & 32 vs. 38 & n.s \\
\hline Karnofsky index $<100 \%$ (yes vs. no) & 16 vs. 77 & 7 vs. 40 & n.s & 13 vs. 57 & 12 vs. 58 & n.s \\
\hline Weight $\operatorname{loss}^{1} \geq 10 \%$ (yes vs. no) & 17 vs. 76 & 11 vs. 36 & n.s. & $10 v s .60$ & 11 vs. 59 & n.s. \\
\hline Neutrophil count $^{1} \geq 5,400$ (yes $v s$. no) & 14 vs. 79 & 8 vs. 39 & n.s. & 10 vs. 60 & 10 vs. 60 & n.s. \\
\hline Lactic dehydrogenase $^{1} \geq 149$ (yes vs. no) & 20 vs. 73 & 8 vs. 39 & n.s. & 15 vs. 55 & 12 vs. 58 & n.s. \\
\hline Smoking history (yes vs. no) & 78 vs. 15 & 39 vs. 8 & n.s. & $61 v s .9$ & 53 vs. 11 & n.s. \\
\hline
\end{tabular}

SD, standard deviation; n.s., not significant.

${ }^{1}$ preoperative determination.

TABLE II - PATIENTS' STRATIFICATION ACCORDING TO RANDOMIZATION AND THE TYPE OF SURGICAL RESECTION

\begin{tabular}{lccr}
\hline \multicolumn{1}{c}{ Resection } & Adjuvant & Control & Total \\
\hline Anatomical & 47 & 46 & 93 \\
Lobectomy & 40 & 35 & 75 \\
Pneumonectomy & 7 & 11 & 18 \\
Minimal & 23 & 24 & 47 \\
Total & 70 & 70 & 140 \\
\hline
\end{tabular}

TABLE III - TOXICITY BY GRADE

\begin{tabular}{lcc}
\hline & \multicolumn{2}{c}{ No. of patients (\%) } \\
\cline { 2 - 3 } & Grade 2 & Grade 3 \\
\hline Hematological & & \\
Leucocytopenia & $30(42)$ & 0 \\
Neutropenia & $25(35)$ & 0 \\
Thrombocytopenia & $8(11)$ & 0 \\
Anaemia & $6(9)$ & 0 \\
Non-Hematological & & 0 \\
Fatigue & $25(35)$ & 0 \\
Flue-like syndrome & $1(1.4)$ & $16(23)$ \\
Nausea/vomiting & $54(77)$ & $2(3)$ \\
Alopecia & $10(18)$ & 0 \\
Neurological symptoms & $5(9)$ & \\
\hline
\end{tabular}

All 140 patients underwent complete surgical resection. As shown in Table II, 47 patients had minimal resection, while 93 underwent anatomical resection. Mean time between surgery and the beginning of chemotherapy was $21 \pm 3.9$ days (range 14-28). Patients were followed up for a mean period of $40.31 \pm 30.86$ months.

Treatment was generally well tolerated, and there was no toxic death. The most adverse effects were manifested starting from the third course. Treatment-related toxicity is reported in Table III. Major discomforts were nausea and frequent vomiting (100\%), fatigue $(35 \%)$, alopecia $(21 \%)$. As regards hematological toxicity, leukopenia was quite relevant (42\% of the patients), especially considering that granulocyte-colony stimulating factors were not routinely available at that time. In most patients, leukopenia rapidly reverted, thus not requiring any dose reduction. Forty-four patients $(63 \%)$ were able to complete the 6 planned courses of the scheduled treatment regimen, whereas 11 patients required a $25 \%$ dose reduction of both drugs (CDDP $75 \mathrm{mg} / \mathrm{m}^{2}$ and VP16 $90 \mathrm{mg}$ / $\mathrm{m}^{2}$ ) due to leukopenia $\mathrm{G} 2$ associated to either thrombocytopenia G2 or emesis. Four additional patients developed a sustained G2 toxicity (over 14 days) at fourth course and stopped treatment thereafter. Seven patients developed rapid disease progression during the chemotherapy and were considered for palliative care only. Finally, 4 patients refused to continue chemotherapy (2 patients after the third course and 2 after the fourth course).

All patients were included in the final survival analyses, regardless their planned treatment was completed or uncompleted, in order to avoid a selection bias leading to an art factual improvement of survival in the adjuvant group. At the end of the study, we
TABLE IV - RECURRENCE PATTERN BY TYPE OF RESECTION AND ADJUVANT TREATMENT

\begin{tabular}{ccccc}
\hline Treatment & None & Local & Distant & Total \\
\hline Minimal & $14(30 \%)$ & $16(34 \%)$ & $17(36 \%)$ & 47 \\
Adjuvant & 11 & 2 & 10 & 23 \\
Control & 3 & 14 & 7 & 24 \\
Anatomical & $46(49 \%)$ & $9(10 \%)$ & $38(41 \%)$ & 93 \\
Adjuvant & 31 & 4 & 12 & 47 \\
Control & 15 & 5 & 26 & 46 \\
\hline
\end{tabular}

recorded a total of 65 deaths, 53 directly of NSCLC and 12 of other unrelated causes. One patient in the adjuvant arm developed a second primary tumor in the contralateral lung. Table IV summarizes the recurrence pattern for treatment and type of resection.

Analysis of DFS time was performed in the overall population. As shown in Figure 2, a significantly higher DFS rate was observed in patients randomized to adjuvant chemotherapy (median survival time 78.4 months) compared to patients randomized to surgery alone (median survival time 25.6 months) $(p=0.0001)$. The 5-year and 10-year DFS rates were 55 versus $20 \%$ and 34 versus $9 \%$, respectively.

Figures 3 and 4 show the Kaplan Meier curves of patients stratified on the basis of different surgical procedure. As shown, in the anatomically resected subset 5-year and 10-year rates for the adjuvant chemotherapy group were 78 and $41 \%$ (median survival time 99.2 months), compared to 30 and $21 \%$ (median survival time 30.4 months) ( $p=0.003$ ) of the control group (Fig. 3). Similarly, in minimally resected patients randomized to adjuvant chemotherapy the 10-year DFS rate was significantly different (38\%) (median survival time 46.4 months) than in patients undergoing minimal resection alone (18\%) (median survival time 24.0 months; $p=0.02$ ) (Fig. 4).

Finally, analysis of overall survival time was performed in the 2 groups of patients. No significant difference was observed according to the type of surgical procedure. Indeed, the 5-year and 10year overall survival rates of anatomically resected patients were 55 and $40 \%$, compared to 40 and $15 \%$ of minimally resected patients ( $p=$ n.s.)(data not shown). On the other hand, a significant difference was observed in the overall survival of patients undergoing adjuvant therapy (median survival time 84.8 months) compared to the control group (median survival time 41.6 months; $p=0.02$ ) (Fig. 5), with 5-year and 10-year survival rates of 62 versus $42 \%$ and 44 versus $20 \%$, respectively. Figure 6 shows the Kaplan Meier overall survival curves for IB stage patients who underwent anatomical resection. As shown, the 5-year and 10-year rates of anatomically resected patients randomized to adjuvant chemotherapy were 75 and $63 \%$, respectively, compared to 50 and $36 \%$ of patients randomized to surgery only (median survival time 49.6 months; $p=0.02$ ) (Fig. 6). Conversely, the 5-year and 10-year survival rates of minimally resected patients randomized to adjuvant chemotherapy were 40 and $20 \%$, respectively, compared to 39 and $18 \%$ of patients randomized to surgery only (Hazard ratio 0.85 , 95\% Confidence Limits: $0.4-1.8, p=0.4$ ). 


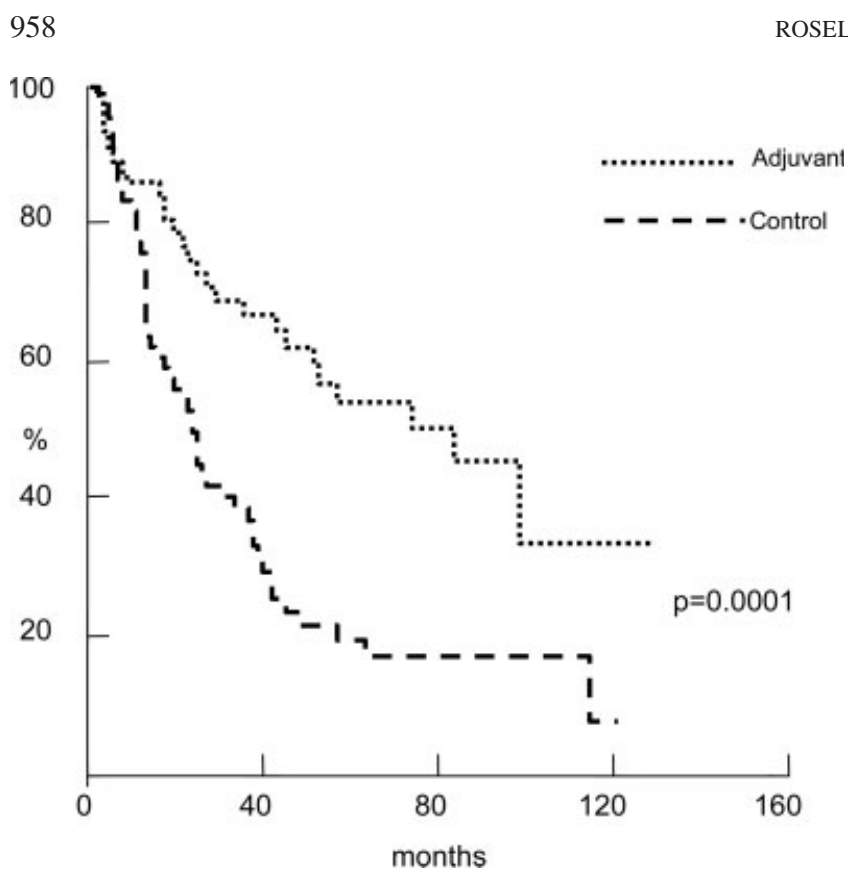

Figure 2 - DFS curves NSCLC stage IB patients (adjuvant $v s$. control).

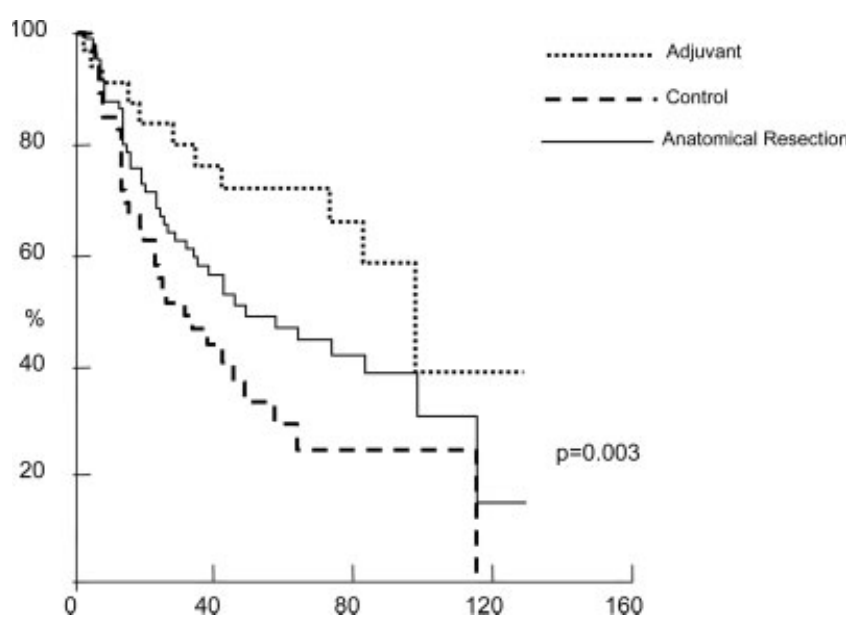

FIgURE 3-DFS curves NSCLC stage IB patients (anatomical resection with/without adjuvant chemotherapy).

\section{Discussion}

Curative surgery can be considered the treatment of choice for early stage NSCLC, although the 5-year survival rates of patients undergoing complete surgical resection is only $40-60 \%{ }^{15,19-21}$

The poor long-term prognosis is due to the development of local and distant recurrences. In fact, distant disease is the most common pattern of failure in stage I NSCLC, and 6-11\% of local recurrence versus $23-30 \%$ of distant metastases is reported in the literature. ${ }^{5,2,22}$ The development of relapsing disease might be attributed to local or systemic undetectable remaining viable tumor cells after resection. ${ }^{5-7}$ For these reasons over the last 15 years the improvement of prognosis by postoperative chemotherapy has been attempted. Accordingly, patients completely resected for stages I and II tumors have been enrolled in adjuvant chemotherapy clinical studies. The cisplatin regimens were the most administered in randomized trials. ${ }^{23}$ Three consecutive studies of the Lung Cancer Study Group (LCSG) testing cyclophosphamide, adriamycin and cisplatin (CAP) regimens in resected NSCLC

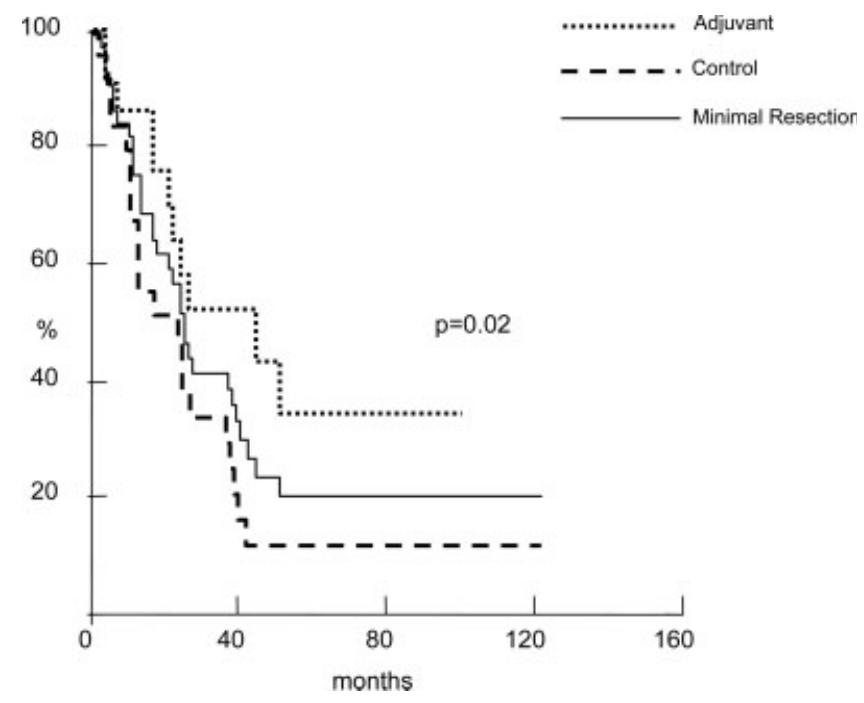

Figure 4 - DFS curves NSCLC stage IB patients (minimal resection with/without adjuvant chemotherapy).

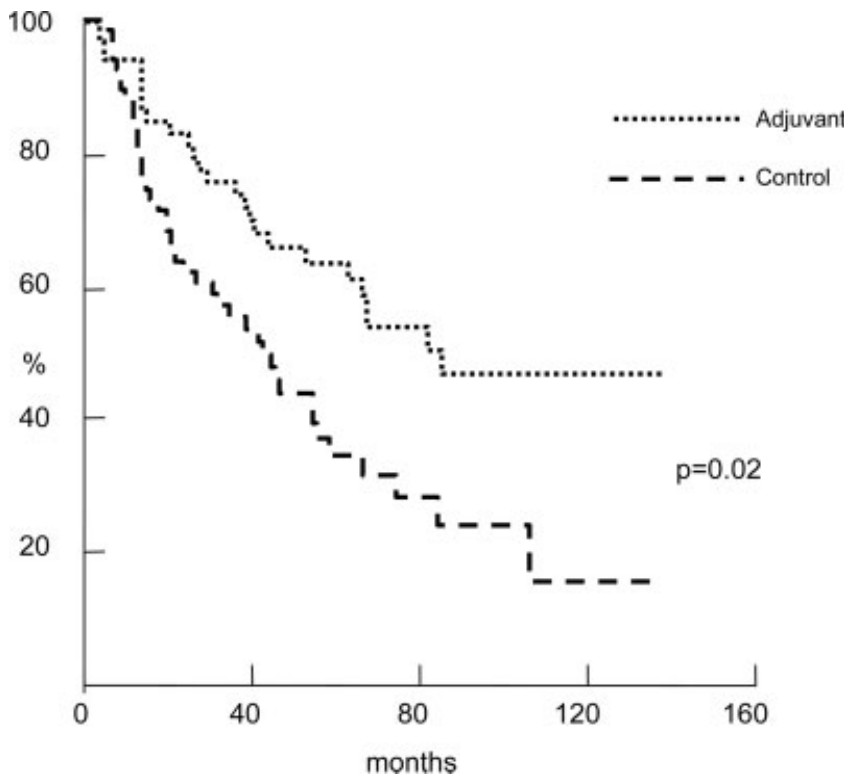

Figure 5-OS curves NSCLC stage IB patients (adjuvant $v s$. control).

patients showed positive biological effects (i.e., delayed recurrence of disease), although overall survival was not significantly different. ${ }^{24-26}$ Moreover, Niiranen et al. in the Finnish Trial ${ }^{8}$ demonstrated a significant improvement in overall survival (56 vs. $67 \%, p=0.05)$ in 110 patients with operated T1-3 N0 by the addition of 6 courses of CAP. On the other hand, 2 other studies, the European Trial ${ }^{27}$ of CAP regimen (administered with a different schedule than the one applied in LCSG trials) and a Japanese trial, ${ }^{28}$ failed to demonstrate any impact on disease-free or overall survival.

For all these reasons, adjuvant chemotherapy in NSCLC cannot be considered as standard therapy, yet, and randomized trials are being presently carried out to assess the real impact on diseasefree or overall survival of NSCLC patients. ${ }^{29,30}$ Preliminary results obtained in these studies are nevertheless divergent. Adjuvant Lung Project Italy study does not show any improvement of either overall survival or DFS. ${ }^{29}$ International Adjuvant Lung Cancer Trial study 


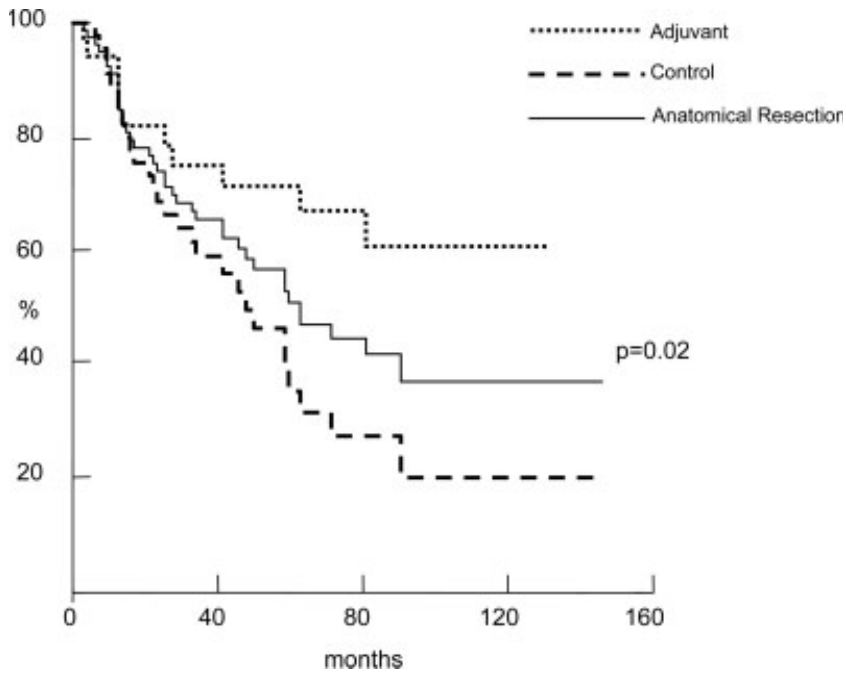

Figure 6 - OS curves NSCLC stage IB patients (anatomical resection with/without adjuvant chemotherapy).

shows an improvement in both parameters, even if limited, in the treated group compared to the control group (overall survival $p<$ 0.03 and DFS $p<0.003){ }^{30}$ Furthermore, the results of other studies $^{31-33}$ demonstrated that presently no agreement has been reached on NSCLC adjuvant therapy.

In a previous trial, we reported that the 5-year overall survival rate of anatomically resected stage IB NSCLC patients who underwent adjuvant chemotherapy was significantly increased $(p=0.04)$. Moreover, the DFS rates were $59 \%$ in patients randomized to adjuvant chemotherapy compared to $30 \%$ in patients randomized to surgery alone $(p=0.02) .{ }^{14}$ In the present study, we have extended the sample size of our previous trial including patients deserving minimal resection and prolonging the postoperative observation period. The results obtained, while confirming the 5-year survival rates obtained in our previous study, demonstrate a significant reduction of the recurrence rates at 10 years, which translates into a long-term survival advantage in stage IB NSCLC patients. Moreover, the patients segmentation according to surgical procedure, allows the identification, within IB stage NSCLC patients, of a subgroup that can derive more advantages from chemotherapy. Indeed, significantly improved overall $(p=0.02)$ and disease-free $(p=0.003)$ survival rates were observed after adjuvant chemotherapy in patients who underwent anatomical resection (Figs. 3 and 6), which is in agreement with the results obtained in recent trials of adjuvant chemotherapy for early stage NSCLC. ${ }^{34-36}$ No statistical difference in overall survival was evident for patients minimally resected, although, a real impact was observed for DFS (Fig. 4). This might be justified by the small size of these subgroups (23 vs. 24). Moreover, we might hypothesize that this effect may be due to the permanence of cancer cells in circulation or hidden in lymphatic organs, which can be responsible for metastases development. ${ }^{6,7}$ In fact, in case of anatomical surgery the residual cell load is probably lower than that occurring after minimal surgery and therefore adjuvant chemotherapy can be more effective.

Limits to our study may be represented by the small sample size due to the strict patients selection. One might also raise some criticism about the type of drugs used at that time. Nonetheless, the results reported here strongly suggest that adjuvant chemotherapy may represent a real impact on long-term survival of stage IB NSCLC by reducing locoregional recurrences and distant metastases rates, particularly in the subset of patients undergoing anatomical resection. This concept is further supported by the results of the CALGB trial (Cancer and Leukemia Group B), demonstrating that adjuvant chemotherapy significantly reduces all cause and lung cancer mortality in stage IB NSCLC. ${ }^{36}$

New trials with new chemotherapic regimens and specifically designed to address this issue are currently ongoing and will help to assess the real impact of adjuvant treatment in the management of NSCLC patients.

\section{Acknowledgements}

This study was performed under the Research Fellowship of Tecnologie e Terapie Avanzate in Chirurgia awarded by Tor Vergata University of Rome.

\section{References}

1. Martini N, Rusch VW, Bains MS, Kris MG, Downey RJ, Flehinger BJ, Ginsberg RJ. Factors influencing ten-year survival in resected stages I to IIIA non-small cell lung cancer. J Thorac Cardiovasc Surg 1999;117: 32-8.

2. Feld R, Rubinstein LV, Weisenberger TH. Site of recurrence stage I non-small-cell lung cancer: a guide for future study. J Clin Oncol 1984; $2: 1352-8$

3. Thomas P, Rubinstein L; Lung Cancer Study Group. Cancer recurrence after resection: T1N0 non-small cell lung cancer. Ann Thorac Surg 1990;49:242-7.

4. Martini N, Bains MS, Burt ME, Zakowki MF, McKormack P, Rusch VW, Ginsberg RJ. Incidence of local recurrence and second primary tumors in resected stage I lung cancer. J Thorac Cardiovasc Surg 1995;109: $120-9$.

5. Matthews MJ, Kanhouwa S, Pickren J, Robinette D. Frequency of residual and metastatic tumor in patients undergoing curative resection for lung cancer, Part 3. Cancer Chemother Rep 1973;4:63-7.

6. Ohgami A, Mitudomi T, Sugio K, Tsuda T, Oyama T, Nishida K, Osaki T, Yasumoto K. Micrometastatic tumor cells in the bone marrow of patients with non-small cell lung cancer. Ann Thorac Surg 1997; 64:363-7.

7. Dobashi K, Sugio K, Osaki T, Oka T, Yasumoto K. Micrometastatic p53-positive cells in the lymph nodes of non-small-cell lung cancer: Prognostic significance. J Thorac Cardiovasc Surg 1997;114:339-46.

8. Niiranen A, Niitamo-Korhonen S, Kouri M, Assendelft A, Mattson K, Pyrhonen S. Adjuvant chemotherapy after radical surgery for nonsmall cell lung cancer: A randomized study. J Clin Oncol 1992;10: 1927-32.

9. Wada H, Miyahara R, Tanaka F, Hitomi S. Postoperative adjuvant chemotherapy with PVM (Cisplatin + Vindesine + Mitomycin C) and UFT (Uracil + Tegaful) in resected stage I-II NSCLC (non-small cell lung cancer): A randomized clinical trial. West Japan Study Group for Lung Cancer Surgery (WJSG). Eur J Cardio-Thorac Surg 1999; $15: 438-43$.

10. NSCLC Collaborative Group. Chemotherapy in non-small cell lung cancer: A meta-analysis using updated data on individual patients from 52 randomised clinical trials. Br Med J 1995;311:899-909.

11. Tonato M. Consensus conference on medical treatment of non-small cell lung cancer. Adjuvant treatment. Lung Cancer 2002;38:S37-S42.

12. Arriagada R, Bergman B, Dunant A, Le Chevalier T, Pignon JP Vansteenkiste J; International Adjuvant Lung Cancer Trial Collaborative Group. Cisplatin-based adjuvant chemotherapy in patients with completely resected non-small-cell lung cancer. N Engl J Med 2004; 350:351-60.

13. Feld R, Rubinstein L, Thomas PA. Adjuvant chemotherapy with cyclophosphamide, doxorubicin, and cisplatin in patients with completely resected stage I non-small cell lung cancer.The Lung Cancer Study Group. J Natl Cancer Inst 1993;85:299-306.

14. Mineo TC, Ambrogi V, Corsaro V, Roselli M. Postoperative adjuvant therapy for stage IB non-small-cell lung cancer. Eur J Card Surg 2001;20:378-84.

15. Naruke $T$, Tsuchiya $R$, Kondo $H$, Hasamura $H$, Nakayama H. Implication of staging in lung cancer. Chest 1997;112:242S-248S

16. Cox DR. Regression models and life tables. J R Stat Soc 1972;34: $187-220$.

17. Kaplan EL, Meier P. Nonparametric estimation from incomplete observations. J Am Stat Assoc 1958;53:457-81.

18. Mantel N. Evaluation of survival data and two new rank order statistics arising in its consideration. Cancer Chemother Rep 1966;50:16370

19. Mountain CF. Revisions in the international system for staging lung cancer. Chest 1997;111:1710-17. 
20. Inoue K, Sato M, Fujimura S, Sakurada A, Takahashi S, Usuda K, Kondo T, Tanita T, Handa M, Saito Y, Sagawa M. Prognostic assessment of 1310 patients with non-small cell lung cancer who underwent complete resection from 1983 to 1993. J Thorac Cardiovasc Surg 1998; 116:407-11.

21. Van Rens MTM, de la Riviere AB, Elbers HRJ, van den Bosch JMM Prognostic assessment of 2,361 patients who underwent pulmonary resection for non-small cell lung cancer, stage I, II, and IIIA. Chest 2000;117:374-9.

22. Pairolero PC, Williams DE, Bergstralh EJ, Piehler JM, Bernatz PE, Payne WS. Post-surgical stage I bronchogenic carcinoma: Morbid implications of recurrent disease. Ann Thorac Surg 1984;38:331-8.

23. Wagner $\mathrm{H}$ Jr. Postoperative adjuvant therapy for patients with resected non-small cell lung cancer: Still controversial after all these years. Chest 2000;117:110S-118S.

24. Holmes EC, Gail M. Surgical adjuvant therapy for stage II and stage III adenocarcinoma and large-cell undifferentiated carcinoma. J Clin Oncol 1986;4:710-5.

25. Lad T. The comparison of CAP chemotherapy and radiotherapy to radiotherapy alone for resected lung cancer with positive margin or involved highest sampled paratracheal node (stage IIIA). LCSG 791. Chest 1994;106:302S-306S.

26. The Lung Cancer Study Group. The benefit of adjuvant treatment for resected locally advanced non-small-cell lung cancer. J Clin Oncol 1988;6:9-17.

27. Dautzenberg B, Chastang C, Arriagada R, Le Chevalier T, Belpomme D, Hurdebourcq M, Lebeau B, Fabre C, Charvoilin P, Guerin RA. Adjuvant radiotherapy versus combined sequential chemotherapy followed by radiotherapy in the treatment of resected non-small-cell lung carcinoma: a randomized trial of 267 patients. Cancer 1995;76:779-86.

28. Ohta M, Tsuchiya R, Shimoyama M, Sawamura K, Mori T, Miyazawa N, Suemasu K, Watanabe Y, Tomita M, Terashima M. Adjuvant chemotherapy for completely resected stage III non-small-cell lung cancer: results of a randomized prospective study.The Japan Clinical Oncology Group. J Thorac Cardiovasc Surg 1993;106:703-8.
29. Scagliotti GU, Fossati R, Torri V, Crino L, Giaccone G, Silvano G, Martelli M, Clerici M, Cognetti F, Tonato M;Adjuvant Lung Project Italy/European Organisation for Research Treatment of Cancer-Lung Cooperative Group Investigators. Randomized study of adjuvant chemotherapy for complete resected I, II, IIIA non small cell lung cancer. J Natl Cancer Inst 2003;95:1453-61.

30. Dunant A, Pignon JP, Le Chevalier T. Adjuvant chemotherapy for non-small cell lung cancer: Contribution of the International Adjuvant Lung Trial. Clin Cancer Res 2005;11:5017s-5021s.

31. Manegold C. Chemotherapy in stage I/II NSCLC and projects of the EORTC - Lung Cancer Group for Early Stage Lung Cancer. Lung Cancer 2001:34:S53-S58.

32. Non-Small Cell Lung Cancer Collaborative Group. Chemotherapy in non-small cell lung cancer: A meta-analysis using updated data on individual patients from 52 randomized clinical trials. Br Med J 1995; 311:899-909.

33. Keller SM, Adak S, Wagner H. A randomized trial of postoperative adjuvant therapy in patients with completely resected stage II or IIIA non-small cell lung cancer. N Engl J Med 2000;343:1217-22.

34. Winton TL, Livingston R, Johnson D, Rigas J, Johnston M, Butts C, Cormier Y, Goss G, Inculet R, Vallieres E, Shepherd F, et al; forNational Cancer Institute of Canada Clinical Trials Group and National Cancer Institute of the United Stated Intergroup JBR. 10 Trial Investigators. Vinorelbine plus cisplatin vs. observation in resected nonsmall-cell lung cancer (NSCLC). N Engl J Med 2005;352:2589-97.

35. Tomek S, End A, Klepetko W. Adjuvant docetaxel plus cisplatin in stage I/II non-small cell lung cancer preliminary results of randomized phase 2 pilot trial of the Central European Cooperative Oncology Group (CECOG). J Clin Oncol 2004;22:7297. [Meeting abstracts.]

36. Strauss GM, Herndon J, Maddaus MA. Randomized clinical trial of adjuvant chemotherapy with paclitaxel and carboplatin following resection in stage IB non-small cell lung cancer (NSCLC): Report of Cancer and Leukemia Group B (CALGB). Protocol 9633. J Clin Oncol 2004; 22:7019. [Meeting abstracts.] 